\title{
Main-ion temperature and plasma rotation measurements based on scattering of electron cyclotron heating waves in ASDEX Upgrade \\ Paper
}

Pedersen, Morten Stejner; Rasmussen, Jesper; Nielsen, Stefan Kragh; Jacobsen, Asger Schou; Korsholm, Søren Bang; Leipold, Frank; McDermott, R. M.; Salewski, Mirko; Schubert, M.; Stober, J. Total number of authors:

11

Published in:

Plasma Physics and Controlled Fusion

Link to article, DOI:

10.1088/1361-6587/aa711a

Publication date:

2017

Document Version

Early version, also known as pre-print

Link back to DTU Orbit

Citation $(A P A)$ :

Pedersen, M. S., Rasmussen, J., Nielsen, S. K., Jacobsen, A. S., Korsholm, S. B., Leipold, F., McDermott, R. M., Salewski, M., Schubert, M., Stober, J., \& Wagner, D. H. (2017). Main-ion temperature and plasma rotation measurements based on scattering of electron cyclotron heating waves in ASDEX Upgrade: Paper. Plasma Physics and Controlled Fusion, 59(7), [075009]. https://doi.org/10.1088/1361-6587/aa711a

\section{General rights}

Copyright and moral rights for the publications made accessible in the public portal are retained by the authors and/or other copyright owners and it is a condition of accessing publications that users recognise and abide by the legal requirements associated with these rights.

- Users may download and print one copy of any publication from the public portal for the purpose of private study or research.

- You may not further distribute the material or use it for any profit-making activity or commercial gain

- You may freely distribute the URL identifying the publication in the public portal 


\section{EUROfusion}

EUROFUSION WPMST1-PR(16) 16174

M Stejner et al.

\section{Main-ion temperature and plasma rotation measurements based on scattering of electron cyclotron heating waves in ASDEX Upgrade}

Preprint of Paper to be submitted for publication in Plasma Physics and Controlled Fusion

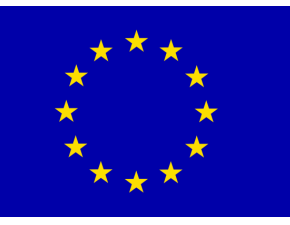

This work has been carried out within the framework of the EUROfusion Consortium and has received funding from the Euratom research and training programme 2014-2018 under grant agreement No 633053 . The views and opinions expressed herein do not necessarily reflect those of the European Commission. 
This document is intended for publication in the open literature. It is made available on the clear understanding that it may not be further circulated and extracts or references may not be published prior to publication of the original when applicable, or without the consent of the Publications Officer, EUROfusion Programme Management Unit, Culham Science Centre, Abingdon, Oxon, OX14 3DB, UK or e-mail Publications.Officer@euro-fusion.org

Enquiries about Copyright and reproduction should be addressed to the Publications Officer, EUROfusion Programme Management Unit, Culham Science Centre, Abingdon, Oxon, OX14 3DB, UK or e-mail Publications.Officer@euro-fusion.org

The contents of this preprint and all other EUROfusion Preprints, Reports and Conference Papers are available to view online free at http://www.euro-fusionscipub.org. This site has full search facilities and e-mail alert options. In the JET specific papers the diagrams contained within the PDFs on this site are hyperlinked 


\title{
Main-ion temperature and plasma rotation measurements based on scattering of electron cyclotron heating waves in ASDEX Upgrade
}

\author{
M. Stejner ${ }^{1}$, J. Rasmussen ${ }^{1}$, S. K. Nielsen ${ }^{1}$, A. S. Jacobsen ${ }^{1}$, \\ S. B. Korsholm ${ }^{1}$, F. Leipold ${ }^{1}$, R. M. McDermott ${ }^{2}$, \\ M. Salewski ${ }^{1}$, M. Schubert ${ }^{2}$, J. Stober ${ }^{2}$, D. H. Wagner ${ }^{2}$, the \\ ASDEX Upgrade Team ${ }^{2}$, and the EUROfusion MST1 Team ${ }^{3}$ \\ ${ }^{1}$ Technical University of Denmark, Department of Physics, DK-2800 Kgs. Lyngby, \\ Denmark \\ ${ }^{2}$ Max-Planck-Institut für Plasmaphysik, Boltzmannstr. 2, 85748 Garching, Germany \\ ${ }^{3}$ See http://www.euro-fusionscipub.org/mst1 \\ E-mail: mspe@fysik.dtu.dk
}

\begin{abstract}
We demonstrate measurements of spectra of $\mathrm{O}$-mode electron cyclotron resonance heating $(\mathrm{ECRH})$ waves scattered collectively from microscopic plasma fluctuations in ASDEX Upgrade discharges with an ITER-like ECRH scenario. The measured spectra are shown to allow determination of the main-ion temperature and plasma rotation velocity. This demonstrates that ECRH systems can be exploited for diagnostic purposes alongside their primary heating purpose in a reactor relevant scenario.
\end{abstract}

PACS numbers: 52.25.Os, 52.35.Hr, 52.40.Db, 52.70.Gw, 52.70.-m 


\section{Introduction}

Measurements of the temperature and rotation velocity of the main fuel-ion population are important to understand and control the behavior of magnetically confined fusion plasmas as these properties directly affect both the plasma confinement and fusion power. However, the high densities and harsh environments foreseen in future fusion power plants, as well as in machines such as W7-X and ITER, will be challenging for most present day measurement techniques. It is therefore relevant to explore alternative diagnostic approaches. Here we will show that the main-ion properties in ASDEX Upgrade (AUG) plasmas can be determined through measurements of scattering of electron cyclotron resonance heating $(\mathrm{ECRH})$ millimeter-waves. As ions move through the plasma, they are screened by the electrons and generate microscopic fluctuations in the plasma density, currents and fields. Scattering of electromagnetic waves off these fluctuations, so-called collective Thomson scattering (CTS), occurs when the length scale corresponding to the wavenumber shift between the incident wave, $\mathbf{k}^{\mathrm{i}}$, and the scattered wave, $\mathbf{k}^{\mathrm{s}}$, is greater than the Debye screening length, $\lambda_{D}$, i.e. when $\left|\mathbf{k}^{\mathrm{s}}-\mathbf{k}^{\mathrm{i}}\right|=k^{\delta} \lesssim 1 / \lambda_{\mathrm{D}}$ (the Salpeter criterion [1]). For millimeter-waves this criterion is easily fulfilled, imposing few restrictions on the scattering geometry, and the spectrum of scattered radiation carries detailed information about the ion velocity distribution. In addition, millimeter-wave systems are generally robust and capable of operating under reactor relevant conditions for extended periods of time with little damage or degradation of performance. Millimeter-wave based CTS, using probing radiation from gyrotrons, is thus a promising diagnostic for confined ion properties with applications including measurements of ion temperatures [2-5], plasma rotation velocities [5], plasma composition $[4,6,7]$, as well as the velocity distribution of nonthermal, fast-ion populations [8-13] and their interactions with plasma instabilities [14-16].

An important aspect of the design of millimeter-wave CTS diagnostics is the ability of the plasma to itself emit and absorb such waves due to the electron cyclotron motion. The background signals in millimeter-wave CTS measurements are typically dominated by electron cyclotron emission (ECE) from the plasma. So, in order to maximize the signal-to-noise ratio, a common approach is to operate at frequencies that avoid any electron cyclotron (EC) resonances along the probe and receiver beam paths. Such designs, avoiding resonances in the plasma, generally allow CTS measurements with sufficient signal-to-noise ratios to resolve the relatively weak fast-ion signatures at large frequency shifts. They are therefore appropriate for measurements of e.g. fast-ion dynamics, which additionally require integration times of no more than a few milliseconds. Thus, the CTS systems at JET [17], TEXTOR [11] and ASDEX Upgrade [18] were designed to operate in O-mode at frequencies between the fundamental and second harmonic electron cyclotron resonances in the plasma. An alternative approach is to operate in X-mode at frequencies below the ECE emission spectrum but above the L-cutoff, as on TFTR [19] and FTU [20,21], and as is intended with the CTS system 
on ITER $[22,23]$.

Here we demonstrate a new approach based on scattering of strongly absorbed electron cyclotron resonance heating $(\mathrm{ECRH})$ radiation in AUG. We conducted measurements with the CTS system during experiments with a heating scheme designed to be qualitatively similar to that of the O1 ECRH scenario in ITER, i.e. with absorption of O-mode gyrotron radiation on the fundamental EC resonance located well inside the plasma. In this regime, the ECE background is far higher than for the normal CTS scenario in AUG. Nevertheless, we find that it is still possible to resolve the thermalion features in the CTS spectra with a signal-to-noise ratio sufficient to allow useful temperature and rotation measurements in good agreement with boron-based charge exchange recombination spectroscopy (CXRS) measurements. CTS based on absorbed probing waves has also been performed in the W7-AS stellarator [3] and in the Large Helical Device [12], but this approach is new for AUG, and here we are further able to show the first detailed comparison between main-ion properties inferred from such measurements and standard impurity-based CXRS measurements. The main advantage of this approach, exploiting scattering of ECRH waves, is that it does not require a dedicated high-power gyrotron as a source of probing radiation. Also, since the probing power is absorbed in the plasma, it presents fewer challenges with respect to reflections and stray probing radiation, which can otherwise give rise to unwanted secondary signal components $[3,13]$ and affect the operation of other microwave diagnostics.

\section{The CTS system at ASDEX Upgrade}

The CTS receiver system on ASDEX Upgrade consists of two heterodyne radiometers, which view the plasma through two of the ECRH transmission lines. The probing radiation is delivered by one of the dual-frequency ECRH gyrotrons. These are normally used in X-mode for second harmonic heating at $140 \mathrm{GHz}$ but were here operated in Omode at $105 \mathrm{GHz}$ to enable studies of the ECRH deposition profile at the fundamental resonance in a scenario similar to that foreseen for the ITER ECRH system. Here the CTS receivers were operated as a piggyback experiment to measure the spectrum of scattered radiation at frequencies around $105 \mathrm{GHz}$. In order to resolve the thermal-ion feature, one of the receivers is equipped with a fast digitizer allowing the frequency range from 104 to $106 \mathrm{GHz}$ to be measured through direct digitization and Fourier analysis of the signal with relatively high frequency resolution (here $1.5 \mathrm{MHz}$ ). The first results from this system as well as its design and operation have been described in some detail elsewhere $[5,24]$.

\section{Plasma conditions}

The measurements presented here were obtained in four H-mode discharges, AUG \#30739, \#30742, \#30746, and \#30749. The plasma current was 1 MA during the flat-top in all four discharges. The magnetic field was $-2.9 \mathrm{~T}$ in the first two and 
$-2.8 \mathrm{~T}$ in the last two discharges. The discharges were otherwise similar, with central densities around $7 \times 10^{19} \mathrm{~m}^{-3}$, NBI heating ramping up to $7 \mathrm{MW}$, and ECRH heating ramping up to 1.2 MW. Time traces for these quantities are shown in Fig. 1. The two ECRH gyrotrons (gyrotron 7 and 8) were modulated in a 50\% duty cycle with on/off periods of $6 \mathrm{~ms}$. From a CTS point of view, gyrotron 7 was used as the probe (i.e. the receiver beam intersected the beam of that gyrotron), and the off-periods were used to measure the ECE background.
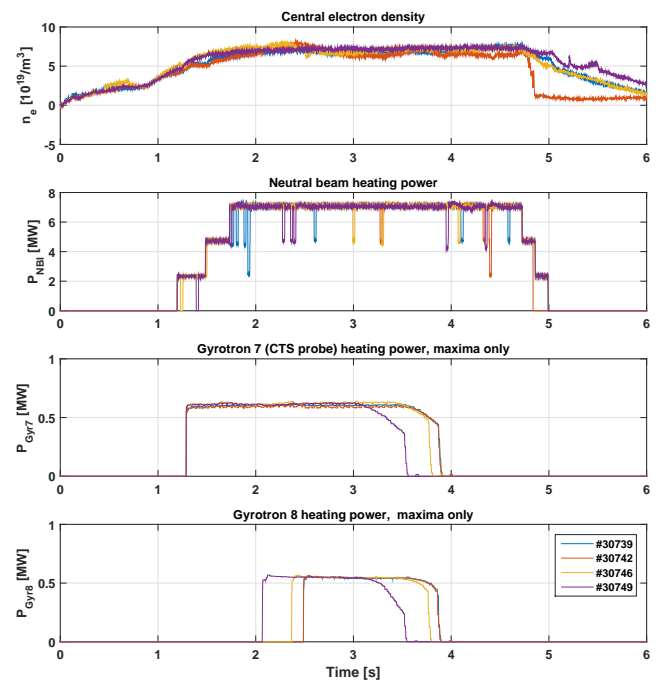

Figure 1. Time traces for the central electron density, total neutral beam injection (NBI) power, and ECRH power from gyrotrons 7 and 8. The gyrotrons were modulated in a $6 \mathrm{~ms}$ on / $6 \mathrm{~ms}$ off duty cycle. The individual modulation cycles would not be distinguishable in the figure, so instead we show the maximum values across a running $12 \mathrm{~ms}$ time interval.

\section{Scattering geometries}

Figure 2 illustrates the CTS beam geometries in these discharges. The beam overlap region, from where the received CTS signal originates, is illustrated by the magenta ellipsoids. In discharge \#30742 the beam overlap was at the position labeled B and was located at $(R, Z)=(1.75 \mathrm{~m},-0.2 \mathrm{~m})$ at normalized poloidal flux-coordinate $\rho_{\mathrm{p}}=0.5$. The scattering angle was $\theta=\angle\left(\mathbf{k}^{\mathrm{i}}, \mathbf{k}^{\mathrm{s}}\right)=128^{\circ}$, and the angle between the resolved fluctuation wave vector and the magnetic field was $\phi=\angle\left(\mathbf{k}^{\delta}, \mathbf{B}\right)=64^{\circ}$. The geometry for discharge \#30746 was very similar, except that the resonance was located a few centimeters further towards the high field side due to the slightly lower magnetic field.

In discharges \#30739 and \#30749 the gyrotron injection angle was the same as in the two other discharges, but the receiver beam was swept along the probe beam to vary the location of the beam overlap region between the positions $\mathrm{A}, \mathrm{B}$ and $\mathrm{C}$ in Fig. 2. Specifically, the measurement position was fixed at $\mathrm{B}$ until $t=2 \mathrm{~s}$. It was 
then swept, first to the high-field side (reaching A at $t=2.38 \mathrm{~s}$ ), and then to the lowfield side (reaching $\mathrm{C}$ at $t=3.2 \mathrm{~s}$ ). It was finally swept back to position $\mathrm{B}$ where it remained from $t=3.6 \mathrm{~s}$ to the end of the discharges. In terms of flux coordinates, the measurement position was thus varied from $\rho_{\mathrm{p}}=0.85$ on the high-field side to $\rho_{\mathrm{p}}=0.65$ on the low-field side. The scattering angle similarly varied between $120^{\circ}$ and $150^{\circ}$ while $\phi$ varied from $62^{\circ}$ to $71^{\circ}$.

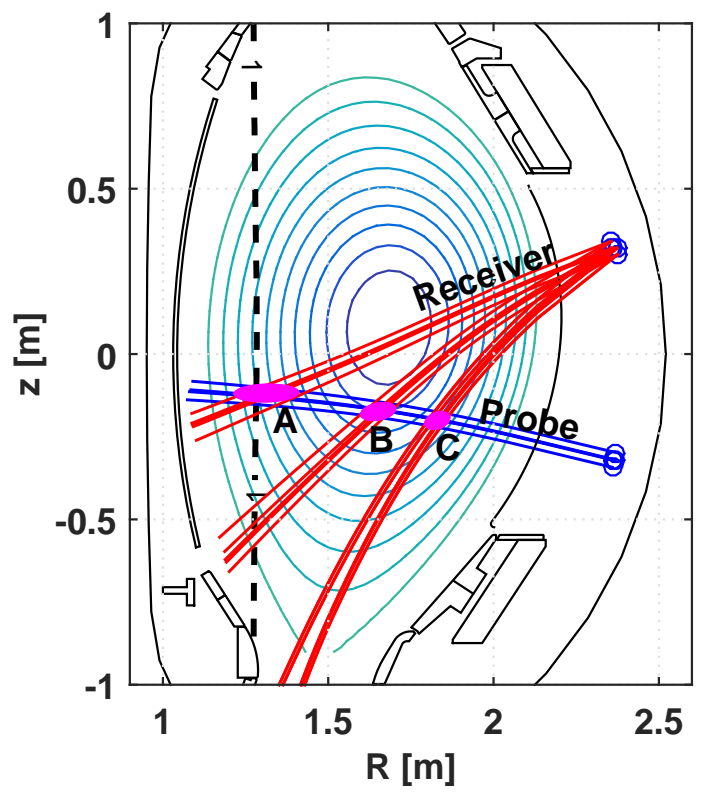

Figure 2. An illustration of the beam geometries in a poloidal projection (calculated with ray tracing). Red: receiver beam at different times. Blue: probe beam. Magenta ellipsoids mark the beam overlap regions. The dashed line marks the fundamental 105 GHz resonance position (this was slightly further out in discharges \#30746 and \#30749 with lower magnetic field). In discharges \#30742 and \#30746 the beam overlap was held at the position labeled B. In discharges \#30739 and \#30749 the receiver beam was swept along the probing beam to move the beam overlap between positions $\mathrm{A}$ and C.

\section{Measured CTS spectra and basic analysis}

The CTS acquisition periods were timed to cover $0.75 \mathrm{~ms}$ before each gyrotron turn-off and $0.7 \mathrm{~ms}$ after. Due to memory and bandwidth limitations, the fast digitizer cannot measure throughout the discharge, so specific acquisition periods must be selected in this manner. Figure 3 shows an example of (uncalibrated) data obtained during one such acquisition period. A number of features are worth pointing out. The gyrotron lines are visible as two fine lines at $104.9 \mathrm{GHz}$ (both gyrotron 7 and 8 were on at this time). To protect the receiver electronics, the frequency range near the gyrotron lines is attenuated by about $100 \mathrm{~dB}$ using two notch filters in series. In the notch filter stop band there is essentially no detectable signal except for the gyrotron lines. At the time when the 
gyrotrons turn off, they chirp quickly towards higher frequencies, potentially ending up outside the notch filter stop band, which would damage the highly sensitive radiometer. To guard against this risk, the signal is further attenuated using a voltage-controlled variable attenuator (VCVA) for $0.3 \mathrm{~ms}$ just after the gyrotron turn-off. During the last part of the acquisition, the gyrotrons are off and the VCVA is open allowing the background signal (mainly ECE) to be measured.

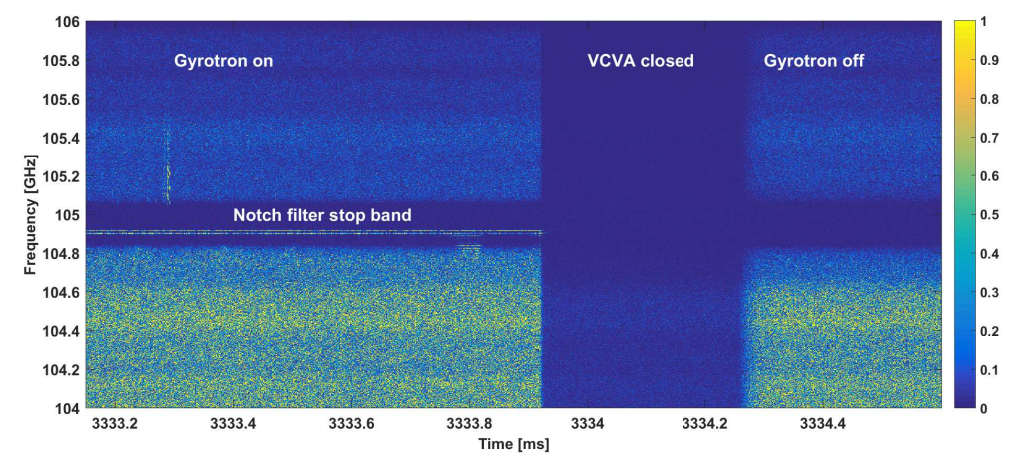

Figure 3. A spectrogram showing uncalibrated CTS data from an acquisition period in AUG \#30739. The gyrotron is on for the first $0.75 \mathrm{~ms}$ of the acquisition period and the gyrotron line is visible inside the notch filter stop band during that period. During and just after the gyrotron turn-off the signal is attenuated by a VCVA for $0.3 \mathrm{~ms}$ and there is then a period with only background measurements.

We note two occurences of very large signal levels (inconsistent with standard CTS signals) during the gyrotron-on period of the acquisition shown in Figure 3. One at $t=3333.3 \mathrm{~ms}$ with a powerful burst of up-shifted signal, and the second at $t=3333.8 \mathrm{~ms}$ where a series of strong, down-shifted lines appears in the spectrogram at frequency intervals of $14 \mathrm{MHz}$. These types of signals occur during only some of the acquisition periods (roughly $1 / 3$ ) in bursts lasting a few $\mu$ s. Usually, only one of the two types found in Fig. 3 occurs during an acquisition period (so, in that respect, Fig. 3 is not a typical example). The origin of these signals is currently unknown, but possible explanations could include effects of parametric decay instabilities $[25,26]$, scattering on transient plasma instabilities, and gyrotron generated noise or secondary modes. However, our present purpose is to analyze the CTS component in the signal, and to that end we just note that these outliers can be rejected as they are clearly identifiable and affect only a fraction of the data during some of the acquisition periods. In principle, one could simply reject every acquisition period affected by such signals, but, to be more economical with the data, we instead seek to remove these burst from within each of the affected acquisition periods using a sigma-clipping procedure: iteratively rejecting positive outliers in each acquisition period until the frequency-specific histogram of the remaining data is consistent with a normal distribution. This has generally been found to be effective in identifying and removing these bursts. The spectra from the affected acquisition periods are then generally consistent with those from the unaffected periods. 


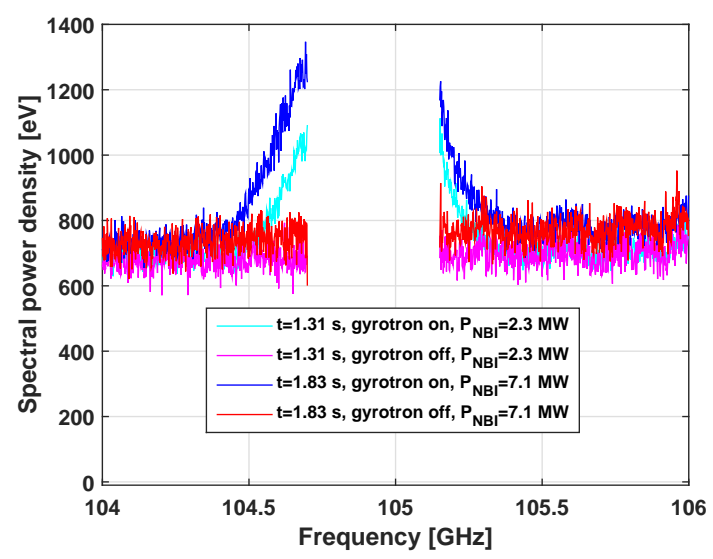

Figure 4. Examples of spectra measured with the CTS system in AUG \#30739 at different NBI power levels and with the probing gyrotron on (blue, cyan) and off (red, magenta), respectively.

To extract the CTS component, we further apply a calibration based on crosscalibrating the ECE background measured during the gyrotron-off periods with electron temperature profiles obtained from integrated data analalysis, IDA [27]. This procedure is similar to the way the TEXTOR CTS system was calibrated [28] and differs from the hot/cold source method usually employed with the CTS system on AUG [24]. In these experiments, with EC resonances for the receiver frequencies well inside the plasma, the ECE background is around $700 \mathrm{eV}, 10-100$ times higher than for the normal CTS operating scenario on AUG. To ensure that these signal levels would not damage the receiver and that the receiver remains in its linear regime, the signal was attenuated by roughly a factor 20 (by partially closing the VCVA mentioned above). The calibration curve obtained with the usual hot/cold source technique would not account for this additional attenuation so we apply the cross-calibration technique. However, the practical difference is limited as the VCVA attenuation is largely independent of frequency and affects mainly the overall scaling of the spectra. The signal within the notch filter stop band cannot be calibrated either way, so that frequency range is excluded in the analysis and the figures presented in the following.

Figure 4 shows two examples of spectra obtained with this procedure in AUG \#30739 at times with different NBI heating powers. The spectra are here shown before background subtraction, and we note that the CTS component is clearly visible above the background at frequencies between 104.5 and $105.5 \mathrm{GHz}$. The spectrum measured at higher NBI power is also clearly seen to be broader, indicating that the effect of higher ion temperature can be discerned despite the relatively high background and noise levels (effects of rotation are less easy to see and require detailed analysis).

For the two examples shown in Fig. 4, the background levels measured at frequencies far from the probing frequency during the gyrotron-on and -off periods are seen to match each other very well. So here the background can easily be subtracted, but this is not always the case. The background can change during an acquisition period, and due 
to the generally high background level this can lead to background subtraction errors significant in comparison to the CTS component. To illustrate this, Fig. 5 shows time traces of the spectral power density obtained after subtracting the background measured during the gyrotron-off periods in AUG \#30739 and binned over frequency intervals of $150 \mathrm{MHz}$. At frequencies far from the probing frequency $(|\delta f|>500 \mathrm{MHz})$, the residuals oscillate around zero, but with outliers ranging up to a few hundred eV. The up- and down-shifted residuals are nearly identical and their variations are highly correlated (linear correlation coefficient above 0.9 between the time traces at $|\delta f|>500 \mathrm{MHz}$ ). The residuals at frequency shifts beyond $500 \mathrm{MHz}$ cannot be explained by variations in the CTS component, which is expected to contribute at most a few eV at such frequencies. Variations in the CTS component would also not explain why the residuals remain the same size after $t=3.9 \mathrm{~s}$ when the probing gyrotron is turned off. At that time the CTS component in the time traces for $|\delta f|<400 \mathrm{MHz}$ disappears and these then show the same residuals as for higher frequency shifts.

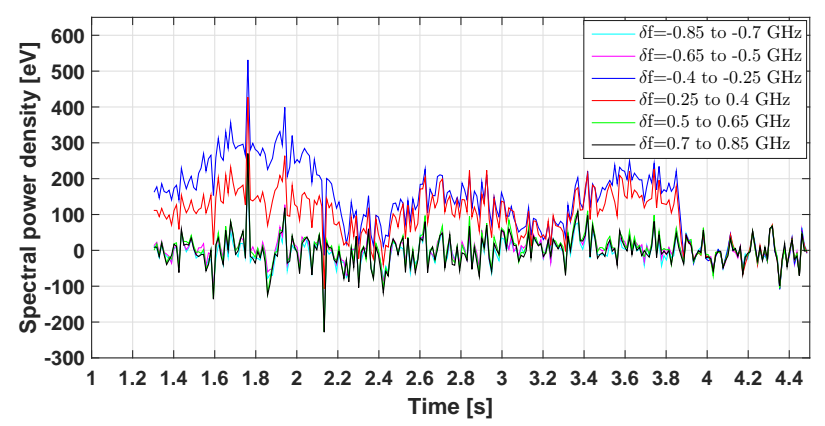

Figure 5. Time traces of the spectral power density found after subtraction of the background signals measured with the probing gyrotron off. The signals are binned over $150 \mathrm{MHz}$ intervals and labeled by their frequency shift relative to the probing gyrotron. The highly correlated residuals at $|\delta f|>500 \mathrm{MHz}$ are best explained by offsets caused by variations in the background within each acquisition period.

The simplest explanation for these residuals is therefore that rapid variations in the background level within each acquisition period lead to errors in the initial background subtraction. These errors are approximately independent of frequency shift and appear as overall offsets in the spectra. We correct for this by subtracting the mean residuals at $|\delta f|>700 \mathrm{MHz}$. This will also subtract any CTS signal present at those frequencies (e.g. due to fast, non-thermal ions), but, as noted above, such signals are expected to be small and the effect will be undetectable considering the statistical noise of roughly 50-60 $\mathrm{eV}$ in these spectra. In hindsight, it might then be argued that the ECE measurements during the gyrotron-off periods were not strictly necessary for background subtraction (which could be based entirely on the signal far from the probing frequency). But, considering that CTS measurements have not been attempted in this scenario before, the ECE measurements are nevertheless important for a complete characterization of the signal. 
In connection with the background subtraction, it is also relevant to consider if the probing radiation itself has an effect on the ECE level [29]. This could potentially cause errors in the background subtraction and distort the CTS spectra, provided the ECRH heating could change the plasma temperature significantly within an acquisition period, or that the temperature could drop significantly during the $0.3 \mathrm{~ms}$ background measurement after the gyrotron turn-off. In particular, one might expect such effects for beam geometries as A in Fig. 2, where the absorption and emission take place on nearby flux surfaces (see Fig. 6). However, the variation of gyrotron power due to the on/off modulation corresponded to less than $10 \%$ of the NBI heating power at the time when measurements were made for geometry A. At other times the gyrotron power variation was larger, but there was little overlap between the locations of the absorption and emission along the probe and receiver beams. So it is reasonable to expect that any effect on the ECE background should be small (in comparison with the CTS component and overall background), and in practice we have not been able to detect any evidence of such effects here. For example, we found no detectable signs of a systematic decrease of the ECE levels consistent with a cooling effect during the background measurement just after the gyrotron turn-off. Thus we conclude such effects are too small to be detected for the noise level in these measurements.

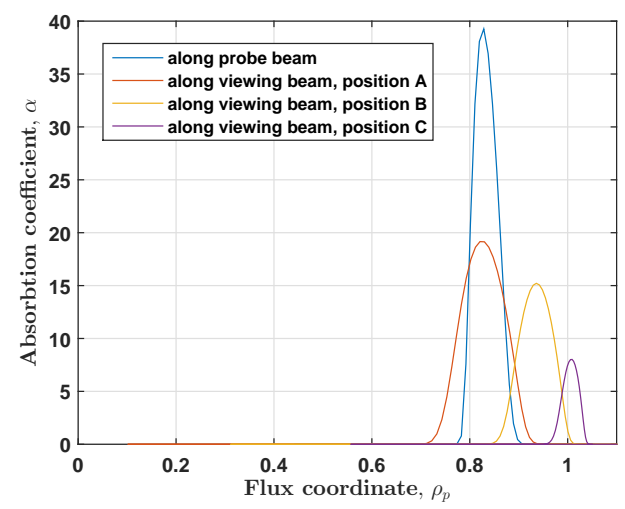

Figure 6. The absorption coefficient calculated along the receiver and probing beam for the beam geometries labeled A, B and C in Fig. 2. For geometry A the absorption and emission happen at the same flux surfaces. For other geometries they are clearly separated.

Figure 7 shows a spectrogram of the CTS measurements obtained through this analysis in discharge \#30739. As mentioned above, the measurement volume was swept along the probing beam in this discharge, covering the radial positions shown in the lower panel. In addition, the NBI power and electron density ramp up gradually until $\mathrm{t}=1.8 \mathrm{~s}$ (see Fig. 1). The spectra in Fig. 7 show a clear response to the resulting changes in the density and temperature in the CTS measurement volume.

As noted above, the observed noise level in these spectra is around 50-60 eV. This is estimated from the standard deviation of the temporal fluctuations of the measured signal within each acquisition period, and it is roughly independent of frequency. The 
signal-to-noise ratio then ranges from zero at large frequency shift, where there is no main-ion CTS signal, to roughly 10 near the notch filter stop band. This corresponds well to the idealized expression for the signal-to-noise ratio in CTS measurements [30], $\mathrm{SNR}=P_{\mathrm{s}}(W T)^{1 / 2} /\left(2\left(P_{\mathrm{s}}+P_{\mathrm{b}}\right)^{2}+2 P_{\mathrm{b}}^{2}\right)^{1 / 2}$, where $P_{\mathrm{s}}$ is the spectral power density of the CTS signal, $P_{\mathrm{b}}$ that of the background, $W$ the frequency resolution and $T$ the integration time. For discharge \#30739, we can insert $P_{\mathrm{s}}<500 \mathrm{eV}, P_{\mathrm{b}}=700 \mathrm{eV}, W=1.5 \mathrm{MHz}$, $T=1.1 \mathrm{~ms}$, and we then get $\mathrm{SNR}<10.3$. This illustrates that, as was also found on TEXTOR [28], the above expression is indeed useful to predict the signal-to-noise ratio in CTS measurements.

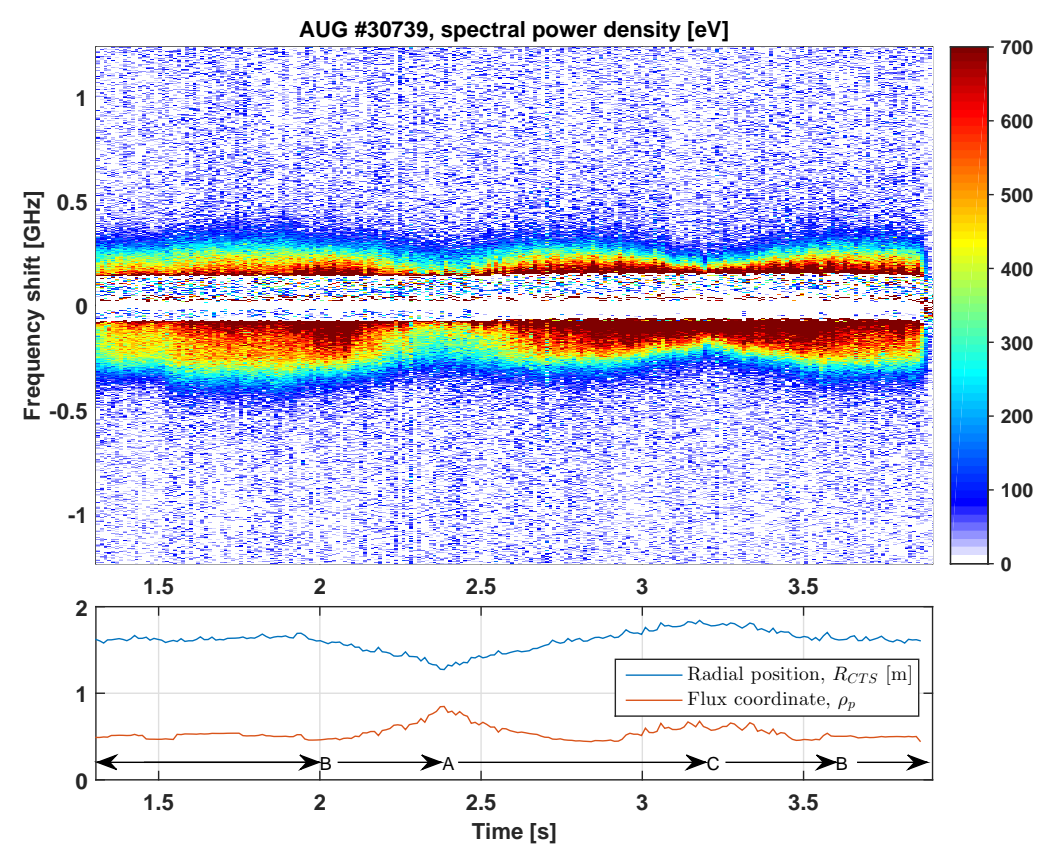

Figure 7. A spectrogram of the CTS measurements in AUG \#30739. In this discharge the measurement position was varied by scanning the receiver beam along the probing beam as explained in Sect. 4. The lower panel shows the radial and the poloidal flux coordinates of the measurement position. The labels A, B and $\mathrm{C}$ refer to the positions defined in Fig. 2. The width and intensity of the measured CTS spectra are seen to vary with the measurement position and with the changes in local plasma conditions.

\section{Results and discussion}

To interpret the CTS measurements and extract information about the ion temperature and plasma rotation, we fit the measured spectra using a model for CTS [31,32] and a least squares fitting method previously used in CTS experiments on TEXTOR and AUG $[4-6,11,15,33,34]$. We here use a simulated-annealing algorithm to minimize the $\chi^{2}$-figure of merit [35]. This approach is comparatively robust against local $\chi^{2}$-minima and variations in the initial guess for fitting parameters and in tests on simulated data it has been found to recover bulk-ion parameters with an accuracy consistent with 
the statistical uncertainty expected for given noise levels in the data $[4,36]$. In the CTS model we assume the ions follow a Maxwellian velocity distribution with plasma rotation described as a drift velocity along the magnetic field. We neglect fast ions as they play a minor role at the frequency shifts considered here, $200 \mathrm{MHz}<|\delta f|<500 \mathrm{MHz}$ ). Other model parameters are fixed at the values measured by other diagnostics and interpolated to the flux coordinate of the CTS measurements. For example, the electron temperature and density are fixed at the values obtained by IDA, and the angles describing the scattering geometry are taken from ray tracing based on input from equilibrium reconstruction and IDA profiles. Uncertainties on these input parameters, as well as on the CTS spectra themselves, were estimated from the temporal fluctuations in each parameter and at each frequency and were taken into account when calculating the statistical uncertainty of the inferred ion temperatures and rotation velocities using a Bayesian framework [33].

Figure 8 shows examples of such fits to spectra measured in AUG \#30739, and Fig. 9 shows comparisons of the ion temperatures and plasma rotation frequencies inferred from the fits to results from boron-based CXRS measurements [37], and to neoclassical TRANSP simulations $[38,39]$ of the main-ion properties (based in part on input from the CXRS measurements).
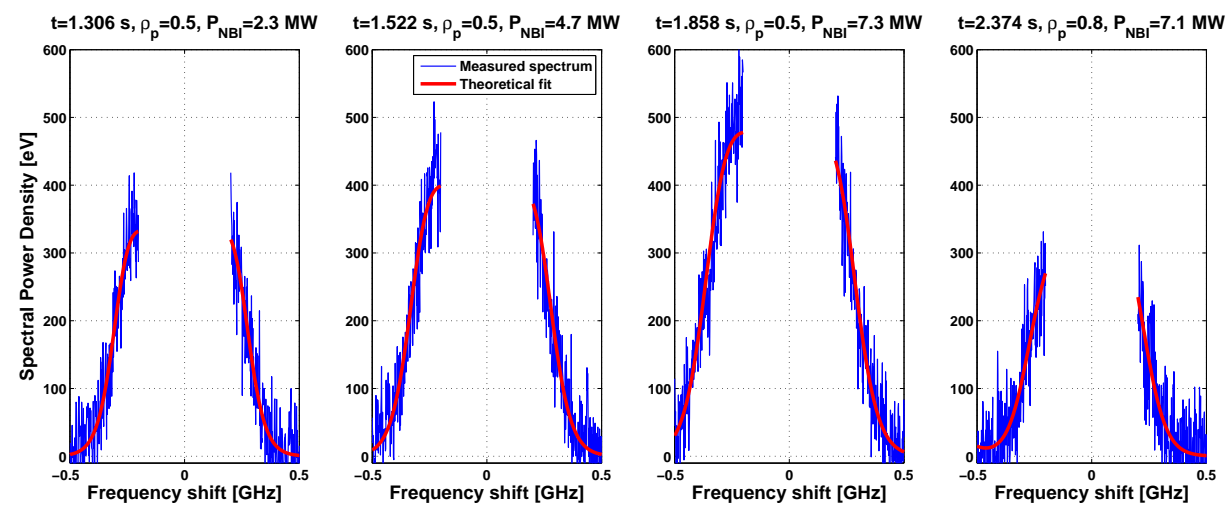

Figure 8. Examples of measured and fitted spectra from AUG \#30739 at different positions and NBI power levels. The example shown to the right was obtained for a position near the EC resonance. Such spectra show no special features, but, as discussed in the text, the fitted model is not valid in that regime, and the ion temperature inferred from the fit is lower than what is measured with CXRS.

In general we find a reasonable level of agreement considering the noise and uncertainties involved in these measurements, but some exceptions are worth mentioning.

In discharges \#30739 and \#30749 the CTS measurement volume was swept along the probing beam. Around $t \simeq 2.38 \mathrm{~s}$ it overlapped with the position of the fundamental EC resonance (the geometry labeled A in Fig. 2, and the example shown furthest to the right in Fig. 8). The CTS model used here assumes [31,40] that the probing frequency is sufficiently far from any EC resonances that the electron velocity distribution is 

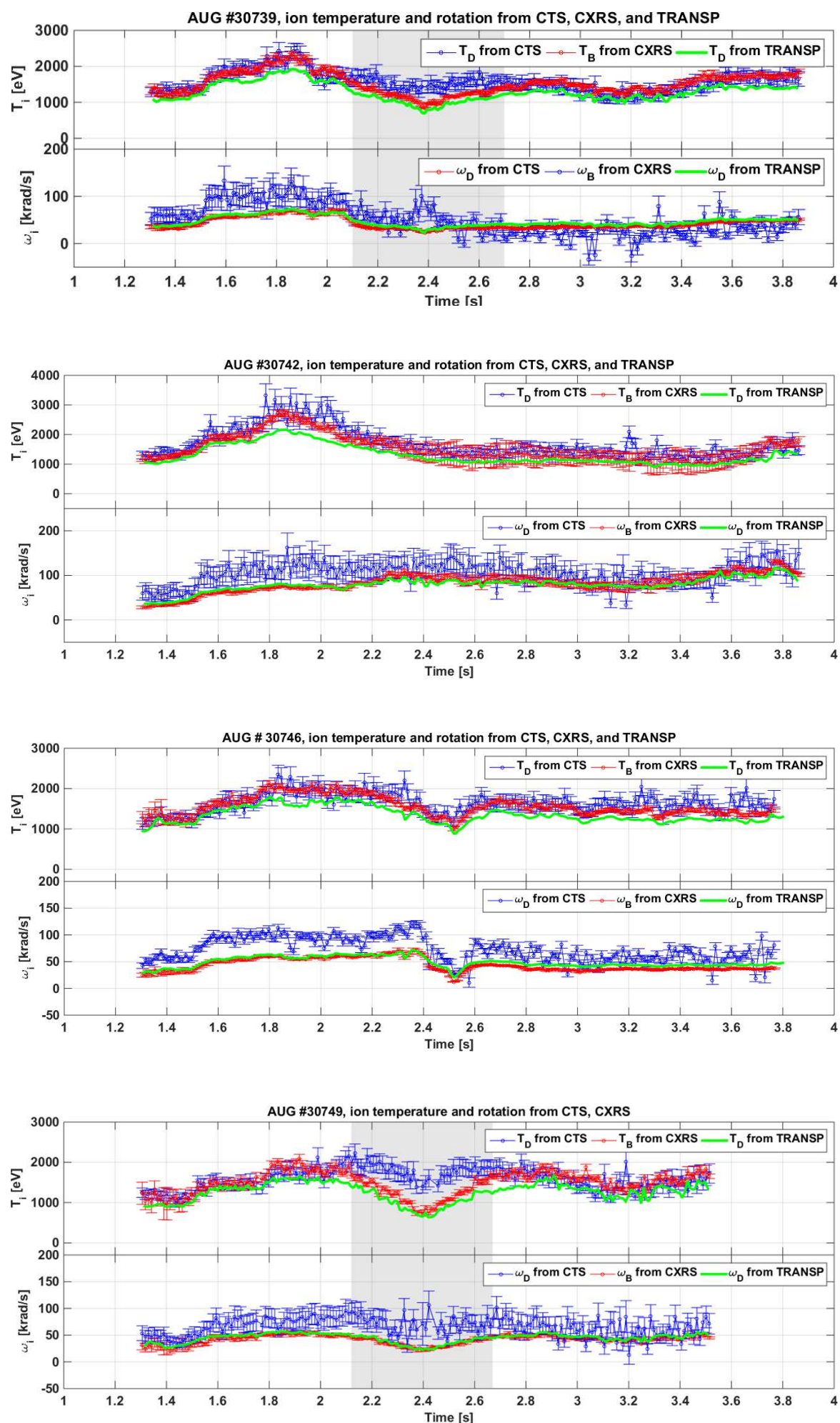

Figure 9. CTS, CXRS and TRANSP results for AUG \#30739, \#30742, \#30746, and \#30749. Upper panels: ion temperatures. Lower panels: rotation frequencies, $\omega_{i}=V_{i} / R$. In discharges \#30739 and \#30749 the measurement position was swept between the two positions labeled A and C in Fig. 2 and covered the coordinates shown in the lower panel of Fig. 7. For those two discharges, the shaded gray areas indicate the time ranges when $v_{\mathrm{Te}} k_{\|}^{\mathrm{s}} /\left(\omega^{\mathrm{s}}-\omega_{\mathrm{c}}\right)>0.12$. In discharges 39742 and 39746 the measurement position was held fixed at the position labeled B in Fig. 2. 
significant only for parallel velocity components, $v_{\|}$, fulfilling $v_{\|} k_{\|}^{\mathrm{s}} /\left(\omega^{\mathrm{s}}-s \omega_{\mathrm{c}}\right) \ll 1$. Here $k_{\|}^{\mathrm{s}}$ is the parallel wave vector component of the scattered waves, $\omega^{\mathrm{s}}$ its angular frequency, $\omega_{\mathrm{c}}$ the angular EC frequency, and $s$ is an integer. Using the electron thermal velocity, $v_{\mathrm{Te}}=\sqrt{2 k_{B} T_{e} / m_{e}}$, for $v_{\|}$and setting $s=1$, Fig. 10 illustrates that this condition is not met in discharges \#30739 and \#30742 around $t \simeq 2.38 \mathrm{~s}$ when the EC frequency in the measurement volume is near the probing frequency. In both discharges the ion temperature measurements by CTS are systematically higher than those from CXRS in the time window $t=2.1-2.7 \mathrm{~s}$. This corresponds to the time when $v_{\mathrm{Te}} k_{\|}^{\mathrm{s}} /\left(\omega^{\mathrm{s}}-\omega_{\mathrm{c}}\right)>0.12$, which may indicate that this is a practical limit for the applicability of the model to O-mode scattering near the fundamental EC resonance. To our knowledge, such a limit has not been experimentally established before. Outside this time window, and in discharges \#30742 and \#30746 with the beam geometry labeled $\mathrm{B}$ in Fig. 2, we stay below this limit. We then find no significant systematic difference between the ion temperatures measured by CTS and CXRS.

The rotation frequencies inferred from the CTS measurements are often somewhat higher than those measured by CXRS in these discharges. These differences are most pronounced during the early part of the discharges, for $t \lesssim 2.5 \mathrm{~s}$, but we have found no clear dependence on plasma parameters or heating scheme. We also note that these differences exist in all four discharges, and that they do not vary systematically with the measurement position or the proximity to the EC resonance as for the ion temperature. Therefore it does not seem likely that this is a result of being near the model limitations with respect to EC resonances.

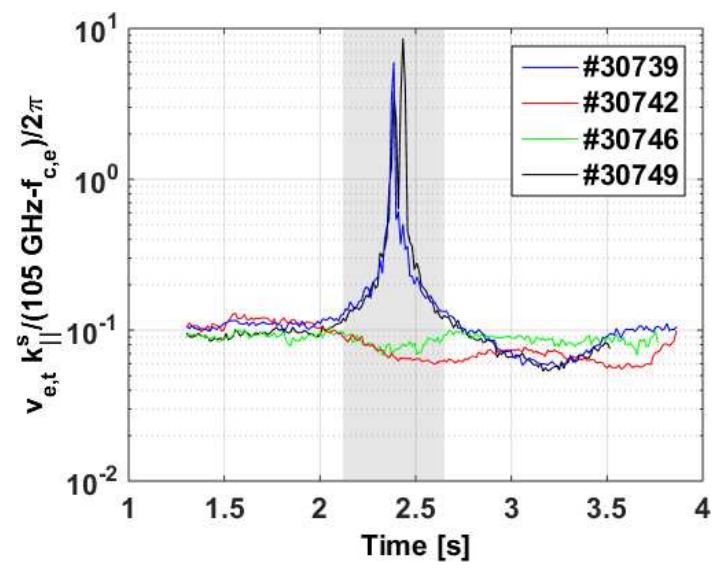

Figure 10. Time traces for the quantity $v_{\|} k_{\|}^{\mathrm{s}} /\left(\omega^{\mathrm{s}}-s \omega_{\mathrm{c}}\right)$ in the CTS measurement volume using the electron thermal velocity for $v_{\|}$and setting $s=1$. The shaded gray area marks the approximate time range where we find significant discrepancies between CTS and CXRS ion temperature measurements in discharges \#30739 and \#30749.

Geometrical effects alone also do not clearly explain these differences. The CTS and CXRS measurements provide ion drift velocities parallel to the magnetic field and in the toroidal direction, respectively. However, the magnetic field is nearly toroidal in the CTS measurement volume, so this is a small effect (less than $1 \%$ correction), 
which does not explain the observed differences. The CTS and CXRS measurements are also made at different positions. We therefore show the measurements as rotation frequencies, and the CXRS measurements are mapped to the time and flux coordinates of the CTS measurements assuming the plasma rotates as a rigid body within each flux surface. Significant poloidal rotation could cause differences between rotation frequencies measured at different locations on the same flux surface [41]. However, the poloidal rotation velocities required to explain the observed differences would be in the range of $5-15 \mathrm{~km} / \mathrm{s}$ while the neoclassical TRANSP simulations indicate deuterium poloidal rotation velocities generally below $5 \mathrm{~km} / \mathrm{s}$. So purely geometric effects and neoclassical poloidal rotation levels are not sufficient to explain the observed differences.

Further experiments will therefore likely be needed to determine the origin of these discrepancies. For the present we note that discrepancies between impurity-based CXRS rotation measurements and Balmer-alpha measurements of the main-ion rotation rates have also been seen in DIII-D [42], as well as in AUG [43] where the deuterium velocity was observed to significantly exceed the nitrogen velocity. In view of these results, we cannot rule out differences between main-ion and impurity rotation rates as the cause of the discrepancies seen here. Balmer-alpha measurements were not available at the time of the experiments presented here, but future benchmarking of CTS and Balmer-alpha measurements could clearly be helpful to determine the cause of these discrepancies.

\section{Conclusion and outlook}

We have shown that scattering of O-mode ECRH waves can be exploited for diagnostic purposes to determine the main-ion temperature and rotation frequency in AUG. In this scenario the ECE background is 10-100 times higher than in the usual CTS scenario on AUG. While this precludes fast-ion measurements, we nevertheless find that signalto-noise ratios up to 10 are achieved for the thermal-ion feature of the CTS spectra using an integration time of $1.45 \mathrm{~ms}$. This is sufficient to allow useful ion temperature measurements, with statistical uncertainties of 8-10\% (around 120-200 eV) and rotation measurements with statistical uncertainties of $20-30 \%$ (around $10-40 \mathrm{~km} / \mathrm{s}$ or $5-25 \mathrm{krad} / \mathrm{s}$ depending on position).

In this scenario, the probing frequency is also closer to the EC resonances in the plasma than usual. This was in some cases found to affect the ion temperature measurements, with discrepancies between CTS and CXRS measurements observed when $v_{\mathrm{Te}} k_{\|}^{\mathrm{s}} /\left(\omega^{\mathrm{s}}-\omega_{\mathrm{c}}\right)>0.12$. The spectra measured above this limit show no special or unexpected features, but they cannot be interpreted with the CTS model used here. Exploitation of scattering closer to the EC resonance would therefore either require more radial launch directions for the probe and receiver beams (to reduce $k_{\|}^{\mathrm{s}}$ ) or further development of the model to accomodate positions near a resonance. For positions further away from the EC resonance, the main-ion temperatures from CTS are generally in good agreement with boron-based CXRS measurements.

The ion rotation frequencies measured by CTS are also comparable to those 
obtained with CXRS, but they do show systematic discrepancies during some phases of the discharges discussed here. These discrepancies do not clearly depend on proximity to the EC resonance, and we have so far been unable to determine their cause, leaving this as a potential topic for future study. One possibility is that they are related to discrepancies observed between impurity- and main-ion based rotation measurements on AUG and DIII-D [42,43].

The measurements presented here were obtained in an ECRH scenario intended to be similar to the O1 heating scenario planned for ITER. Hence it seems pertinent to comment briefly on the possibility of such measurements there. Fig. 11 illustrates an example of how this might work on a conceptual level. We have here assumed that $170 \mathrm{GHz}$ ECRH waves launched from an equatorial port plug can be viewed with a receiver system located in an upper port plug. For simplicity, the waves are further assumed to be launched radially in the same poloidal plane. It should be noted that the present design for the ITER ECRH system [44,45] foresees large toroidal injection angles with no steering capability in the toroidal direction and with the launchers displaced toroidally. So Fig. 11 should be viewed only as a conceptual illustration, and more detailed calculations would be required to assess if main-ion CTS measurements would require a dedicated receiver system or could be incorporated in the ECRH system as on AUG.

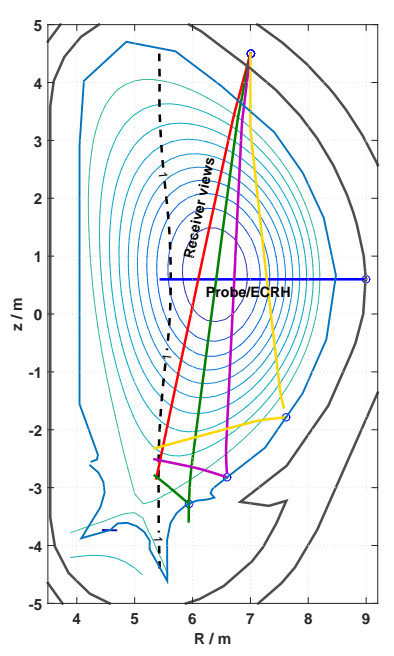

Figure 11. Ray tracing for a $170 \mathrm{GHz}$ CTS geometry in ITER assuming a standard plasma shape in the ITER baseline sccenario with $B_{T}=-5.3 \mathrm{~T}$. The probe (ECRH wave) is assumed to be launched radially from an equatorial port and viewed from an upper port. As there is presently no detailed design for such a system, we have no estimate of the beam width, and we therefore show only a single ray for the probe and four examples of receiver rays with different injection angles. The measurements would be localized near the intersections of the probe and receiver rays. All rays terminate near the (cold) fundamental $170 \mathrm{GHz}$ EC resonance indicated by the thick dashed line, either directly or through reflections off the plasma facing wall. 
To minimize the ECE background, the receiver lines of sight in Fig. 11 are terminated on the fundamental resonance in the (relatively) cold part of the plasma around $\rho_{\mathrm{p}}=0.95-0.97$. The resonance is reached directly for the leftmost (red) viewing direction, and through reflections (assumed to be specular) on the plasma facing wall for measurement positions further on the low field side (green, purple, and yellow lines). Such an approach was considered in some detail for the fast-ion CTS diagnostic in ITER [46]. It was ultimately rejected (in favor of a $60 \mathrm{GHz}$ option) because the spectral power density of the ECE background could not be reduced below $3.5 \mathrm{keV}$, which would not allow useful fast-ion measurements. Nevertheless, it may still be possible to achieve a signal-to-noise ratio sufficient to exploit scattering of the ECRH waves for main-ion measurements. For the parameters used in the fast-ion feasibility study [46] the spectral power density of the thermal feature in the CTS spectra ranged up to $\sim 100 \mathrm{eV}$. Assuming a background of $3.5 \mathrm{keV}$, the signal-to-noise ratio would then be around 10, as in the present experiments, for a frequency resolution of $10 \mathrm{MHz}$ and a temporal resolution of $50 \mathrm{~ms}$. While a more detailed assessment is beyond our scope here, we note that such simple signal-to-noise considerations therefore would seem to allow exploitation of scattered ECRH waves for main-ion diagnostics. However, it should also be noted that, at the leftmost beam intersection in Fig. 11 (red receiver view), we find that $v_{\mathrm{Te}} k_{\|}^{\mathrm{s}} /\left(\omega^{\mathrm{s}}-\omega_{\mathrm{c}}\right)=0.11$, i.e. just around the limit established here. Assuming this limit also applies under ITER conditions, interpretation of measurements closer to the resonance or for larger $k_{\|}^{\mathrm{s}}$ would then again depend on new developments in CTS theory.

Finally, we note that most present machines usually employ ECRH in X-mode at a second harmonic EC resonance, and that this can in principle also be exploited for diagnostic purposes. The CTS system on AUG has recently been equipped with a $140 \mathrm{GHz}$ receiver line, which, among other applications, will be used to test this proposal. Early results from this system indicate that it is indeed possible to detect and interpret CTS spectra of a quality useful for main-ion measurements in a second harmonic X-mode heating scenario as well [47].

\section{Acknowledgments}

This work has been carried out within the framework of the EUROfusion Consortium and has received funding from the Euratom research and training programme 20142018 under grant agreement No 633053. The views and opinions expressed herein do not necessarily reflect those of the European Commission.

\section{References}

[1] E. Salpeter. Physical Review, 120(5):1528-1535, dec 1960.

[2] E. V. Suvorov, et al. Plasma Physics and Controlled Fusion, 37(11):1207-1213, 1995.

[3] E V Suvorov, et al. Plasma Physics and Controlled Fusion, 39(12B):B337-B351, dec 1997.

[4] M Stejner, et al. Plasma Physics and Controlled Fusion, 55(8):085002, aug 2013. 
[5] M Stejner, et al. Plasma Physics and Controlled Fusion, 57(6):062001, jun 2015.

[6] M. Stejner, et al. Plasma Physics and Controlled Fusion, 54(1):015008, jan 2012.

[7] M. Stejner, et al. The Review of scientific instruments, 83(10):10E307, oct 2012.

[8] H. Bindslev, et al. Physical Review Letters, 83(16):3206-3209, oct 1999.

[9] H. Bindslev, et al. Physical Review Letters, 97(20):205005, nov 2006.

[10] M. Salewski, et al. Nuclear Fusion, 50(3):035012, mar 2010.

[11] S. K. Nielsen, et al. Physical Review E, 77(1):016407-11, jan 2008.

[12] M. Nishiura, et al. Nuclear Fusion, 54(2):023006, feb 2014.

[13] S K Nielsen, et al. Plasma Physics and Controlled Fusion, 57(3):035009, mar 2015.

[14] S. K. Nielsen, et al. Nuclear Fusion, 51(6):063014, jun 2011.

[15] S. K. Nielsen, et al. Plasma Physics and Controlled Fusion, 52(9):092001, sep 2010.

[16] J. Rasmussen, et al. Nuclear Fusion, 56(11):112014, nov 2016.

[17] J. A. Hoekzema, et al. Review of Scientific Instruments, 68(1):275, jan 1997.

[18] F. Meo, et al. The Review of scientific instruments, 79(10):10E501, oct 2008.

[19] John S. Machuzak, et al. Review of Scientific Instruments, 68(1):458, 1997.

[20] U. Tartari, et al. Nuclear Fusion, 46(11):928-940, nov 2006.

[21] W. Bin, et al. Fusion Engineering and Design, 96-97:733-737, oct 2015.

[22] H. Bindslev, et al. ITER Fast Ion Collective Thomson Scattering, Detailed integrated design of the collective Thomson scattering (CTS) system for ITER. Technical report, http://orbit.dtu.dk/en/publications/iter-fast-ion-collective-thomson-scattering(220dc911ddcb-43a5-9f96-c7a2a0153f86).html, 2005.

[23] F. Meo, H. Bindslev, and S. B. Korsholm. ITER fast ion collective Thomson scattering, Conceptual design of $60 \mathrm{GHz}$ system, Ris $\varnothing-\mathrm{R}-1600(\mathrm{EN})$. Technical report, http://www.orbit.dtu.dk/en/publications/iter-fast-ion-collective-thomson-scatteringconceptual-design-of-60-ghz-system(30e4aab5-22c1-43e0-91a1-39f12584b782).html, 2007.

[24] M. Stejner, et al. Review of Scientific Instruments, 85(9):093504, sep 2014.

[25] E. Gusakov and A. Popov. Physical Review Letters, 105(11):115003, sep 2010.

[26] S K Nielsen, et al. Plasma Physics and Controlled Fusion, 55(11):115003, nov 2013.

[27] R. Fischer, et al. Fusion Science and Technology, 58(2):675-684, oct 2010.

[28] M. Stejner, et al. Review of Scientific Instruments, 81(10):10D515, 2010.

[29] S. Kubo, et al. The Review of scientific instruments, 81(10):10D535, oct 2010.

[30] H. Bindslev. On the theory of Thomson scattering and reflectometry in a relativistic magnetized plasma. Phd. thesis, Ris $\varnothing$ National Laboratory, Roskilde, Denmark, Ris $\varnothing-R-663,1992$.

[31] H. Bindslev. Plasma Physics and Controlled Fusion, 35(11):1615-1640, 1993.

[32] H. Bindslev. J. Atmos. Terr. Phys., 58:983-989, 1996.

[33] H. Bindslev. Review of Scientific Instruments, 70(1):1093, 1999.

[34] J Rasmussen, et al. Plasma Physics and Controlled Fusion, 57(7):075014, jul 2015.

[35] W. H. Press, et al. Numerical recipes in FORTRAN. The art of scientific computing. Cambridge: University Press, 1992.

[36] M. Stejner, et al. Nuclear Fusion, 52(2):023011, feb 2012.

[37] E. Viezzer, et al. Review of Scientific Instruments, 83(10):103501, 2012.

[38] A. Pankin, et al. Computer Physics Communications, 159(3):157-184, jun 2004.

[39] W. A. Houlberg, et al. Physics of Plasmas, 4(9):3230, sep 1997.

[40] H. Bindslev. JET-P(95)16: Relativistic effects in millimeter wave applications on magnetically confined fusion plasmas. Technical report, 1995.

[41] Per Helander and Dieter J. Sigmar. Collisional transport in magnetized plasmas. Cambridge University Press, 2002.

[42] B. A. Grierson, et al. Physics of Plasmas, 19(5):056107, mar 2012.

[43] B. Geiger, et al. Measurement and interpretation of active Balmer Alpha spectra at ASDEX Upgrade. In Proc. of the 42th EPS Conference on Plasma Physics, page P1.116, 2015.

[44] M. Henderson, et al. Physics of Plasmas, 22(2):021808, feb 2015. 
[45] T. Omori, et al. Fusion Engineering and Design, 86(6-8):951-954, oct 2011.

[46] H. Bindslev, F. Meo, and S. B. Korsholm. ITER Fast Ion Collective Thomson Scattering Feasibility study. Technical report, http://orbit.dtu.dk/en/publications/iter-fast-ion-collectivethomson-scattering(401e562c-65a3-4345-9032-da5cc8b9013b).html, 2003.

[47] M Stejner, et al. Submitted to IRMMW-THz2016, 2016. 